\title{
EL BICAMERALISMO EN EL SIGLO XXI. LOS ÚLTIMOS DEBATES SOBRE EL SENADO EN EL DERECHO COMPARADO
}

\author{
EVA SÁENZ ROYO \\ Profesora Titular (A) de Derecho Constitucional \\ Universidad de Zaragoza
}

\begin{abstract}
SUMARIO
I. El declive del bicameralismo de finales del siglo xx y su repunte a comienzos del siglo XxI. II. El reciente debate sobre el Senado en los Estados Unitarios: los casos de Reino Unido, Francia e Irlanda. III. El reciente debate sobre el Senado en los Estados Federales y descentralizados políticamente: los casos de Canadá, Australia, Italia y Bélgica. IV. Consideraciones finales.
\end{abstract}

\section{EL DECLIVE DEL BICAMERALISMO DE FINALES DEL SIGLO XX Y SU REPUNTE A COMIENZOS DEL SIGLO XXI}

El bicameralismo se vincula históricamente con la altamente estratificada sociedad de la Edad Media y refleja «el espíritu comunal del mundo medieval» ${ }^{1}$. El origen del mismo se suele situar en el Reino Unido en el siglo XIV (1339) cuando los Commons comenzaron a reunirse con el clero bajo separadamente de los Peers fuera de la estructura del parlamento medieval ${ }^{2}$. Pero su surgimiento lejos de ser un diseño deliberado que responda a un modelo filosófico concreto, es más bien un accidente histórico ${ }^{3}$. Un desarrollo semejante se produjo en Hungría (1397) o en

1 Patterson, S. C./Mughan, A., «Senates and the Theory of Bicameralism», Patterson, S. C./ Mughan, A. (ed.), Senates. Bicameralism in the contemporany world, Ohio State University Press/Columbus, 1999 , p. 2.

2 LUTHER, J., "The search for a constitutional geography and historiography of second chambers», Luther, J./Passaglia, P./Tarchi, R. (eds.),A world of second chambers: Handbook for constitutional studies on bicameralism, Giuffré Editore, 2006, pp. 8-9; De Cueto Nogueras, C., «Política y Gobierno en el Reino Unido: ¿Ante el agotamiento del ciclo de revisionismo constitucional laborista?, De CuETo, C./Durán, M., Regímenes políticos contemporáneos. Entre inmovilismo y cambio, Comares, 2008, p. 60.

3 Patterson, S. C./Mughan, A., «Senates and the Theory of Bicameralism», ob. cit., pp. 2-3. 
Polonia (1453), aunque el bicameralismo no fue el único modelo de orden en la Europa medieval. La mayoría de los territorios del Imperio carolingio adoptaron la fórmula tricameral o, incluso, cuatro cámaras hubo en Dinamarca o en Suecia ${ }^{4}$.

No obstante, desde que la Revolución Francesa y Americana atribuyeran el poder soberano al conjunto de la comunidad y se configurara el gobierno representativo como única forma de organizar el ejercicio del poder político, parecía difícil sostener que el interés general de una sociedad pudiera ser representado en dos cámaras. Desde el más puro liberalismo, si la nación es una, su voluntad no puede ser sino única y su representación ha de hacerse en un único órgano ${ }^{5}$. El repetido argumento atribuido al abate Sièyes es contundente: la voluntad general no puede ser más que una en cada momento; por consiguiente, el poder legislativo no debe corresponder a dos Cámaras, pues si ambas están de acuerdo, una de ellas es inútil, y, en caso contrario, la Cámara no representativa debe desaparecer ${ }^{6}$. O, en palabras posteriores de Jeremy Bentham, «si una segunda cámara representa el interés general, ésta es inútil; y si representa un interés particular, es maliciosa» ${ }^{7}$.

La fuerza de los hechos, sin embargo, con una sociedad fuertemente desigual, convirtió al bicameralismo también en liberal, "concebido entonces como un subproducto necesario, expresión jurídico-política del onmipresente principio de división de los poderes, imprescindible obstáculo para garantizar un moderado comportamiento del Parlamento» ${ }^{8}$.

Sólo la extensión del sufragio y la progresiva asunción del principio democrático de la soberanía popular harán palmaria la contradicción entre el bicameralismo y la concepción unitaria del pueblo. A partir de ese momento, «(l)a voluntad general legitimada por el principio mayoritario se adecúa mal a la ideafuerza liberal de limitación del poder. El bicameralismo colisiona con la democracia gobernante, pues sólo la elección da carácter representativo a los órganos constitucionales» ${ }^{9}$. Desde esta perspectiva, la segunda cámara no puede ser sino en «anomalía» ${ }^{10}$ y su supresión parecería ineludible.

4 Luther, J., «The search for a constitutional geography and historiography of second chambers», ob. cit., pp. 8-9.

5 Fiorentino, K., «Histoire intelectuelle d'un débat éternel», Pouvoirs. Le Sénat pour quoi faire?, 159, 2016, p. 20.

6 En realidad, Sieyès no fue un irredento opositor del bicameralismo. Al principio apoyó la idea de una única cámara que luego se dividiera a efectos prácticos. A mediados de 1790 apoyó el bicameralismo convencional, e incluso se convirtió en presidente del senado conservador napoleónico en 1799. Vid. LuTHER, J., «The search for a constitutional geography and historiography of second chambers», ob. cit., pp. 12-13.

7 Rockow, L., «Bentham on the Theory of Second Chambers», The American Political Science Review, 22, 3, 1928, pp. 577-578.

8 Chueca Rodríguez, R., «Teoría y práctica del bicameralismo en la Constitución Española», REDC, 10, 1984, pp. 63-64. p. 67.

9 Chueca Rodríguez, R., «Teoría y práctica del bicameralismo en la Constitución Española», ob. cit.,

10 Término ahora común entre los franceses desde que así lo definiera el ex primer ministro Lionel Jospin en 2005. Vid. Fiorentino, K., «Histoire intelectuelle d'un débat éternel», ob. cit., p. 15. 
Y esta parece ser la tendencia dominante en el siglo Xx. El primer parlamento unicameral democrático en Europa se remonta a 1906 en Finlandia. A partir de ese momento la representación del pueblo en una única cámara progresó rápidamente a comienzos del siglo Xx. El declive del bicameralismo durante el siglo xx fue claro. Mientras el bicameralismo representaba el 59\% en 1961 pasó a suponer el $46 \%$ en 1976 , el $34 \%$ en 1986 y el $33 \%$ en 1996 . Este declive responde a la aparición de nuevos países con parlamentos unicamerales y a la abolición de la segunda cámara por parte de un total de 31 países, entre ellos Nueva Zelanda en 1951, Dinamarca en 1953 o Suecia en $1970^{11}$. Una excepción a esta tendencia han sido en el siglo xx los Estados Federales, aunque sí se ha tendido a suprimir la segunda cámara en el ámbito subestatal ${ }^{12}$.

A comienzos del siglo xxI se produce, en contra de la tendencia generalizada en el siglo xx, un repunte del bicameralismo. Desde 1996 hasta 2014 son 28 los países que adoptan una segunda cámara, frente a sólo 9 que la suprimen. Curiosamente, como subraya Coakley, este repunte del bicameralismo de comienzos del siglo XXI tiene poco que ver con la expansión del federalismo. De los 28 países que crean o restablecen esa segunda cámara desde 1996, sólo uno de ellos responde al modelo federal. Mientras que en 1996 el bicameralismo estaba presente en el $82 \%$ de los Estados Federales, solo en el $26 \%$ de los Estados Unitarios, en el 2014 baja el porcentaje en los Estados Federales al $76 \%$ y sube al $35 \%$ en los Estados unitarios ${ }^{13}$.

Sin embargo, la tendencia aparentemente inversa al siglo xx no lo es tanto si tomamos en consideración que los veintisiete nuevos Estados unitarios bicamerales creados desde 1996 no responden a los parámetros democráticos. Por tanto, «aunque la tendencia a la baja del bicameralismo se ha parado y, al menos a corto plazo, se ha invertido, no hay evidencia de que representen ningún tipo de expansión de las fronteras de la democracia liberal» ${ }^{14}$.

Democracia y bicameralismo siguen constituyendo en el siglo XXI dos términos conflictivos de difícil encaje. Actualmente, de los veintiocho países que conforman la UE, quince tienen un parlamento unicameral: Bulgaria, Chipre, Croacia, Dinamarca, Estonia, Finlandia, Grecia, Hungría, Letonia, Lituania, Luxemburgo, Malta, Portugal, Eslovaquia y Suecia. Por otra parte, siguen siendo

11 CoAkley, J., «The Strange Revival of Bicameralism», The Journal of Legislative Studies, 20, 4, 2014. En línea: http://www.tandfonline.com/doi/full/10.1080/13572334.2014.926168?src=recsys (consultado el 16.12.2016).

12 Han optado por el unicameralismo las provincias de Canadá, los estados de Brasil, los länder en Alemania o los cantones suizos. Vid. MAssicotTe, L., «Legislative unicameralism: A global survey and a few case studies», Journal of Legislative Studies, 7, 1, 2001, pp. 153-154. En línea: http://www.tandfonline.com/ doi/10.1080/714003865 (consultado el 11.01.2017).

13 CoAKley, J., «The Strange Revival of Bicameralism», ob. cit.

14 CoAkley, J., «The Strange Revival of Bicameralism», ob. cit. 
Estados unitarios bicamerales el Reino Unido, Francia, Irlanda, República checa o Eslovenia ${ }^{15}$.

A continuación analizaremos los últimos debates que sobre el Senado se han desarrollado en algunas de las democracias contemporáneas. Veremos cómo mientras en los Estados unitarios se reproducen los debates ya vividos en el siglo Xx, en los Estados federales se observa un nuevo cuestionamiento de la segunda cámara y una tendencia también hacia su reducción o, incluso, supresión. Veamos.

\section{EL RECIENTE DEBATE SOBRE EL SENADO EN LOS ESTADOS UNITARIOS: LOS CASOS DE REINO UNIDO, FRANCIA E IRLANDA}

Cada vez que se plantea el bicameralismo en los Estados unitarios se vuelve al dilema planteado por Sieyès. Si la segunda cámara es de representación política y tiene poder real, duplica el poder de la primera cámara. En este caso, si está de acuerdo con la primera es redundante y si no lo está es maliciosa, ya que no puede contrariar la voluntad general expresada ya por la primera de las Cámaras. Si la segunda cámara es de representación política, pero sin poder real o claramente subordinado a la primera, también sería redundante. Pero si es una cámara que no es de representación política de la voluntad general, sino que representa intereses particulares como pueden ser los colectivos resistentes a los cambios y que hacen valer su tradicional autoridad para legitimar su posición (esto ha justificado el bicameralismo en nuestro constitucionalismo histórico), o representando determinados colectivos en los que se estructura la sociedad y que potencialmente pueden aportar al debate nacional (intelectuales, grupos desfavorecidos, minorías étnicas, religiosas o lingüísticas, sindicatos o asociaciones empresariales...), tiene también difícil encaje en las democracias contemporáneas en cuanto la voluntad general se entiende única y formada a través de la suma de las voluntades individuales del conjunto de los ciudadanos.

Frente a la contundencia de estos argumentos, dos son básicamente las justificaciones que se han dado para el mantenimiento de las segundas cámaras en los Estados unitarios.

Una reproduce la metáfora utilizada por Washington para responder a la pregunta de Jefferson sobre la necesidad de una segunda Cámara en la Constitución americana ${ }^{16}$ : como cámara de enfriamiento de las pasiones, de reflexión y, en

15 Benetti, J., «Et si le Sénat n'existait pas?», Pouvoirs. Le Sénat pour quoi faire?, 159, 2016, p. 7-8.

16 Cuando Jefferson le pregunta a Washington por qué se ha introducido el Senado en la Constitución americana, Washington le pregunta: ¿Por qué viertes tu café en el plato? Jefferson replica: «Para enfriarlo». Washington le responde que «Precisamente por eso. Vertimos la legislación en el plato senatorial para enfriarlo». Conversación reproducida en Patterson, S. C./Mughan, A., «Senates and the Theory of Bicameralism», ob. cit., p. 15 . 
definitiva y a la larga, de mejora de la técnica legislativa. No obstante, la calidad legislativa de los sistemas bicamerales no ha probado ser superior a la de los sistemas monocamerales. El monocameralismo escandinavo viene a demostrarlo. Que exista una única cámara no impide que la ley pueda ser sometida a varias lecturas «de enfriamiento» (tres en Dinamarca — art. 41 de su constitución-o en Islandia — art. 44 de su Constitución- $)^{17}$, con lo que se logra un procedimiento más pausado y, si se quiere, una mejora de la calidad técnica. Además, la función de mejora de la calidad legislativa no exige de una cámara que participe de la función legislativa, sino que basta con un órgano más bien de carácter asesor.

En segundo lugar, es habitual señalar que la segunda cámara puede corregir los posibles excesos mayoritarios de la primera cámara, favoreciendo así el check and balance, en el sentido defendido tempranamente por Montesquieu ${ }^{18}$ o en $E l$ Federalista ${ }^{19}$ y más tarde por Stuart Mill ${ }^{20}$. Sin embargo, los excesos del principio mayoritario se salvan con otros instrumentos mucho más eficaces - y menos contradictorios - en las democracias contemporáneas como son las fórmulas electorales proporcionales, las mayorías cualificadas, el referéndum legislativo de iniciativa popular o el control de la constitucionalidad de las leyes ${ }^{21}$.

A pesar de la contundencia de los argumentos, el bicameralismo pervive en algunas democracias con organización territorial unitaria. La contradicción de esta práctica con la contundencia de la teoría ha provocado en estos Estados un debate eterno, que llega hasta nuestros días, y en el que siguen reiterándose los mismos argumentos para tratar de justificar su supervivencia. A continuación veremos las últimas reformas y debates en los tres Estados unitarios más significativos de Europa.

\section{a) El caso del Reino Unido}

A pesar del proceso de descentralización política del Reino Unido (devolution), de jure sigue considerándose un Estado Unitario y la razón de ser del bicameralismo sigue respondiendo a esta forma jurídica de Estado.

17 Benetti, J., «Et si le Sénat n'existait pas?», ob. cit., pp. 9-10.

18 Montesquieu, Del Espíritu de las Leyes, Tecnos, Madrid, 1993, L. XI, C. VI, pp. 110 y 113.

19 Hamilton o Madison LXII, Hamilton/Madison/Jay, El federalista, Fondo de Cultura Económica, 2014, pp. 263-264.

20 MILL, J. S., Del Gobierno representativo, Tecnos, Madrid, 1985, pp. 148-154.

21 En este mismo sentido, Massicotte, L., «Legislative unicameralism: A global survey and a few case studies», ob. cit., p. 165; Torres Del Moral, A., «El Parlamento: monocameralismo y bicameralismo», Ius et Praxis, 25, 1995, p. 37; Chueca Rodríguez, R., «Teoría y práctica del bicameralismo en la Constitución Española», ob. cit., p. 72; Ruíz Ruíz, J. J., «Más dependiente y menos competente: el nuevo senado belga de comunidades y regiones tras la reforma constitucional», Teoría y realidad constitucional, 38, 2016, p. 373. 
Como ya hemos señalado, la segunda cámara tiene su origen en la Inglaterra medieval, siendo las más antigua de Europa, y también allí aparece tempranamente el debate sobre su necesaria supresión. El 19 de marzo de 1649, la Cámara de los Lores fue abolida por un Acta del Parlamento que declaraba que «los Comunes de Inglaterra declaran, tras una larga experiencia, la inutilidad de la Cámara de los Lores y su peligro para el pueblo de Inglaterra». Pero tras el fracaso de Cromwell en la abolición de esta cámara, se validó el bicameralismo moderno ${ }^{22}$.

La Cámara de los Lores se volvió a reunir en 1660, convirtiéndose de nuevo en la Cámara más poderosa del Parlamento, posición que ocuparía hasta el siglo XIX. No obstante, la progresiva democratización del país conllevaría irremediablemente a la reducción progresiva de su poder. La Cámara de los Comunes comenzó a adquirir mayor importancia sobre todo a raíz de la reforma electoral de 1832 y los poderes legislativos de la Cámara de los Lores serían drásticamente reducidos durante el siglo xx. La Parliament Act 1911 excluía a la Cámara de los Lores de la tramitación de las leyes financieras y presupuestarias y reducía a dos años su capacidad de veto suspensivo para el resto de las leyes. En 1949 se aprobó una nueva Parliament Act que redujo el veto suspensivo de los Lores a únicamente un año. A partir de ese momento, su incapacidad para vetar una ley más allá de un año la convierte fundamentalmente en cámara de consulta y reflexión. Con la Life Peerages Act 1958 se crean los Lores vitalicios (miembros no hereditarios de la Cámara), acabando así con la exclusiva naturaleza hereditaria del cargo y permitiendo la inclusión en la Cámara de los Lores de mujeres, de miembros meritocráticos (dentro de los cuales estarían los Lores Espirituales que son obispos elegidos por su prestigio y dilatada carrera eclesiástica dentro de la Iglesia anglicana) y de miembros procedentes de partidos políticos. En 1963 la Life Peerages Act también permitió que los Lores hereditarios pudieran renunciar a su título. La House of Lord Act (1999) pretende la eliminación de los Lores hereditarios, si bien de forma interina siguen existiendo 92 que son elegidos entre los aristócra$\operatorname{tas}^{23}$. Actualmente solo hay dos lores auténticamente hereditarios. En fin, tras la entrada en funcionamiento el primero de octubre de 2009 de la nueva Corte Suprema del Reino Unido, este órgano asumió la función judicial que hasta entonces ostentaba la Cámara Alta a través del Appellate Committee.

En definitiva, una historia de reformas progresivas que en la práctica han llevado a convertir su carácter aristocrático en otro más meritocrático, a la vez que reducir su función a la meramente consultiva y deliberativa. De hecho, su función de control es claramente secundaria, ya que no puede comprometer la responsabilidad del gobierno, y la función legislativa está claramente subordinada a la Cámara de los Comunes. Desempeña una labor deliberativa importante respecto

22 Luther, J., «The search for a constitutional geography and historiography of second chambers», ob. cit., p. 10 .

23 Vid. De Cueto Nogueras, C., «Política y Gobierno en el Reino Unido: ¿Ante el agotamiento del ciclo de revisionismo constitucional laborista?, ob. cit., pp. 66-71. 
a las leyes no controvertidas y especialmente complejas técnicamente, ya que estas leyes tienen una primera audiencia en esta cámara para pasar luego los Comunes. También ha desempeñado una función importante de mejora técnica y de detalle de las leyes aprobadas en los Comunes ${ }^{24}$. Pero su capacidad de veto de las leyes aprobadas en la Cámara de los Comunes puede ser fácilmente superada por ésta con un nuevo posicionamiento en el siguiente período de sesiones. En todo caso, la supervivencia durante tanto tiempo de esta institución históricamente anacrónica sólo puede explicarse por la extrema cautela en la utilización de sus poderes residuales y la perfecta consciencia de que cualquier otra actitud «hubiese sido suicida» ${ }^{25}$.

\section{b) El caso de Francia}

La supervivencia del Senado francés también resulta del todo paradójica, siendo que en ningún otro Estado las críticas hacia el mismo han sido tan crudas. Desde la Revolución francesa, cuando el bicameralismo se consideró contra-revolucionario ${ }^{26}$, hasta las recientes calificaciones del Senado como «anomalía democrática» ${ }^{27}$, con peticiones recientes de supresión desde las más altas instancias ${ }^{28}$. $\mathrm{Y}$ a pesar de las feroces críticas, el bicameralismo pervive en Francia.

El Senado francés es elegido por aproximadamente 150.000 «grandes electores», entre los que se encuentran los diputados parlamentarios, los diputados provinciales, los diputados regionales y los concejales de ayuntamiento. La forma de elección da una mayor importancia a la representación del mundo rural. No obstante, se trata de un bicameralismo imperfecto por cuanto el Gobierno es únicamente responsable ante la Asamblea Nacional y, salvo el caso de la revisión constitucional, el Gobierno puede disponer que los diputados parlamentarios tengan la última palabra en la aprobación de las leyes ${ }^{29}$.

24 Vid. Ahumada, M., «Informes sobre segundas cámaras en Europa: la Cámara de los Lores», Fundación Manuel Giménez Abad. En línea: http://www2.congreso.gob.pe/sicr/cendocbib/con3_uibd.nsf/ C7E4E6132A711DAF052579AC007BB9DD/\$FILE/6.INFORMESSOBRELAC\%C3\%81MARADELOSLORESMarianAhumada.pdf (consultado el 16.01.2017).

25 De Cueto Nogueras, C., «Política y Gobierno en el Reino Unido: ¿Ante el agotamiento del ciclo de revisionismo constitucional laborista?, ob. cit., p. 70.

26 Fiorentino, K., «Histoire intelectuelle d'un débat éternel», ob. cit., p. 18.

27 Término ahora común entre los franceses desde que así lo definiera el ex primer ministro Lionel Jospin en 2005. Vid. La entrevista de Le Monde a Lionel Jospin el 21 de abril de 1998. En línea. http://discours. vie-publique.fr/notices/983001223.html (consultado el 16.01.2017). FiORENTINO, K., «Histoire intelectuelle d'un débat éternel», ob. cit., p. 15.

28 Claude Bartolone, presidente socialista de la Asamblea Nacional, el 28 de enero de 2015 se pronunció en un plató de televisión "por la desaparición del Senado» en su forma actual. Vid. Le Figaro, 29 de enero de 2015. En línea: http://www.lefigaro.fr/politique/le-scan/couacs/2015/01/29/25005-20150129ARTFIG00292entre-les-presidents-du-senat-et-de-l-assemblee-la-guerre-est-declaree.php (consultado el 16.01.2017).

29 Peres, H., «Francia: la larga duración del presidencialismo flexible», De Cueto, C./Durán, M., Regímenes políticos contemporáneos. Entre inmovilismo y cambio, Comares, 2008, p. 155-156. 
El Senado francés ni cumple la función de representación territorial (es un Estado unitario) ni de representación local, sino que la representación sigue siendo de la nación entera ${ }^{30}$. Los argumentos actuales para su supervivencia siguen girando en torno a su función como cámara de reflexión y de mejora de la calidad legislativa ${ }^{31}$ o como un instrumento de contrapoder en un presidencialismo mayoritario ${ }^{32}$. No obstante, siguen siendo diversas las propuestas para su supresión o conversión, o bien en una cámara al servicio de la descentralización, o en una cámara corporativa de representación de intereses económicos, familiares e intelectuales ${ }^{33}$. En octubre de 2015 un grupo de trabajo constituido por la Asamblea Nacional sobre el futuro de las instituciones propuso su fusión con el Consejo económico, social y medioambiental y su conversión básicamente en cámara de técnica legislativa y consultiva ${ }^{34}$.

c) El caso de Irlanda

El referéndum irlandés sobre la supresión del senado del 4 de octubre de 2013 es también buena muestra del debate actual sobre la supervivencia de esta institución en este Estado unitario.

La constitución irlandesa de 1937 estableció un sistema bicameral en el que la segunda cámara (Seanad Éireann) era en parte indirectamente elegida y en parte nombrada, pero sobre todo claramente subordinada a la primera cámara. Ésta designa al Presidente del Gobierno y aprueba su Gobierno, que es responsable ante el Dáil y debe contar con la confianza de la mayoría. El Senado sólo posee un débil poder de enmendar o retrasar un proyecto de ley, poder que utiliza en pocas ocasiones. Además, de los 60 miembros del Senado Irlandés, once senadores son designados libremente por el Presidente del Gobierno y cuarenta y nueve elegidos indirectamente: seis por ciertas Universidades y el resto de entre personas con conocimientos y experiencia en cinco sectores profesionales (cultura, agricultura, trabajo, industria y comercio y administración pública), por los miembros de la Cámara de Representantes nueva, los del Senado saliente y los

30 BENETTI, J., «Et si le Sénat n'existait pas?», ob. cit., p. 8; LE YonCourT, T., «Le Sénat républicain représentant des collectivités territoriales?, Pouvoirs. Le Sénat pour quoi faire?, 159, 2016, pp. 38-39.

31 Vid. TÜRK, P., «Le Sénat: une assemblée de bons légistes?», Pouvoirs. Le Sénat pour quoi faire?, 159, 2016, pp. 65-78.

32 BENETTI, J., «Et si le Sénat n'existait pas?», ob. cit., p. 8; LE YonCOURT, T., «Le Sénat républicain représentant des collectivités territoriales?, ob. cit., pp. 5-14. Sobre el papel del Senado y sus relaciones con el Presidente de la República vid. De SAINT SERnin, J., «La majorité sénatoriale sous la Vº́publique». Les différentes configurations à l'égard du pouvoir exécutif», Pouvoirs. Le Sénat pour quoi faire?, 159, 2016, pp. 53-64.

33 Sobre las diversas propuestas puestas sobre la mesa vid. Fiorentino, K., «Histoire intelectuelle d'un débat éternel», ob. cit., p. 16.

34 Refaire la démocratie, Groupe de Travail sur l'avenir des institutions, octubre de 2015, pp. 102-105. En línea: http://www.ladocumentationfrancaise.fr/var/storage/rapports-publics/154000692.pdf (consultado el 17.01.2017). 
representantes de los entes locales ${ }^{35}$. No obstante, al ser elegidos la mayor parte (43) por colegios electorales dominados por consejeros locales se convierte en realidad en otra cámara que «representa intereses partidistas» ${ }^{36}$.

La pervivencia del Senado se ha mantenido a pesar de que su función y su composición han sido objeto de continua controversia durante el siglo Xx. Una pervivencia que parecía estar llamada a su fin a comienzos del siglo XXI. Unos meses después de que Enda Kenny, el líder del Fine Gael, abogara por la reforma de la segunda cámara, en octubre de 2009 sorpresivamente anunció su intención de abogar por un sistema unicameral. Esta propuesta tomó forma en el documento 'New Politics' que publicó el partido en marzo de 2010 y posteriormente en su programa electoral en 2011. Para entonces también el Labour Party y el Sinn Féin había llegado a la conclusión de la necesidad de suprimir el Senado. En 2011 el Fianna Fáil no mantenía una posición tan absoluta y de hecho se opusieron, junto al Green Party, a su supresión durante la campaña de referéndum proponiendo una reforma absoluta de la institución (A Seanad for the people). Tras las elecciones de 2011, el programa de gobierno de la coalición entre el Labour Party y el Fine Gael incorporó la abolición del Senado, que exigiría una reforma constitucional aprobada parlamentariamente y la subsiguiente celebración de un referéndum ${ }^{37}$.

Sorprendentemente, tras la aprobación parlamentaria, con una participación del $39.17 \%$ y por un margen de 42.500 votos, la propuesta de suprimir el senado en referéndum fue rechazada por un $51.7 \%$. El principal argumento esgrimido para su supresión (y el que más pesó en el electorado) fue el coste que suponía su mantenimiento, aunque también se argumentó su carácter no democrático, su falta de utilidad y su carácter esencialmente partidista, así como su conversión en «lugar de retiro» de los políticos. Por otro lado, el argumento que más pesó para su mantenimiento fue la función de contrapoder al gobierno.

Actualmente la única propuesta de reforma del Senado se refiere a los seis senadores elegidos entre los graduados universitarios. La redacción constitucional original del art. 18 preveía que tres senadores fueran elegidos por los graduados universitarios de la Universidad Nacional y otros tres por los de la Universidad de Dublín (Trinity College), las dos únicas Universidades que existían cuando la Constitución se aprobó. La séptima enmienda constitucional, de 1.979, permite que por ley se puedan considerar otras Universidades e instituciones de enseñanza superior como circunscripción electoral, pero, hasta

35 Rey Martínez, F., «El peculiar Senado irlandés», Fundación Manuel Giménez Abad. En línea: http://www.fundacionmgimenezabad.es/images/Documentos/2005/20051205_ot_rey_martinez_f_es_o.pdf (consultado el 24.01.2017).

36 COAKLEy, J., «The Strange Revival of Bicameralism», ob. cit.

37 Maccarthaight, M./ Martin, S. M., «Bicameralism in the Republic of Ireland: The Seanad Abolition Referendum», Irish Political Studies, 30, 1, 2015, pp. 121-131. En línea: http://dx.doi.org/10.108 0/07907184.2014.981534 (consultado el 19.01.2017). 
la fecha, ninguna ley se ha dictado en este sentido. La propuesta de reforma consistiría en aprobar una ley que permita que todas las Universidades se constituyan en circunscripción electoral. Esto supondría una ampliación del electorado de 151.000 a casi 800.000 personas, lo cual puede fortalecer el carácter ya muy partidista del Senado, ya que la necesidad de más fuentes y medios para la campaña exigirá del apoyo de los partidos políticos también para los candidatos universitarios $^{38}$.

\section{EL RECIENTE DEBATE SOBRE EL SENADO EN LOS ESTADOS FEDERALES Y DESCENTRALIZADOS POLÍTICAMENTE: LOS CASOS DE CANADÁ, AUSTRALIA, ITALIA Y BÉLGICA}

Desde que Madison en 1788 señalara el bicameralismo como uno de los rasgos que definían a la nueva organización del Estado que surgía con la Constitución americana, ha sido constante en la doctrina ver en este bicameralismo una esencia de todo Estado Federal. Un bicameralismo justificado, según Madison, en la distinta representación que ostentarían ambas Cámaras: La Cámara de Representantes derivará sus poderes del pueblo de América, y el pueblo estará representado en la misma proporción y con arreglo al mismo principio que en la legislatura de un Estado particular. Hasta aquí el gobierno es nacional y no federal. En cambio, el Senado recibirá su poder de los Estados, como sociedades políticas y coiguales, y estas estarán representadas en el Senado conforme al criterio de igualdad, como lo están ahora en el actual Congreso. Hasta aquí el gobierno es federal y no nacional $^{39}$.

Sin olvidar que en los Estados de vieja tradición democrática los instituciones son fruto de sus particulares historias nacionales ${ }^{40}$, esta idea de la representación «de las partes» en un Senado frente a la representación del pueblo en un Congreso cundió en la formación de todos los Estados Federales y constituye sin duda en su origen un rasgo distintivo del Estado federal frente a otras formas de organización territorial del poder.

De hecho, tal y como señala Patrice Gélard en su informe sobre Les Secondes Chambres en Europe de la Comisión de Venecia, el bicameralismo está unido al federalismo y la descentralización política ya que «la segunda cámara parece tener como vocación primordial asegurar la representación de colectividades

38 Maccarthaight, M./ Martin, S. M., «Bicameralism in the Republic of Ireland: The Seanad Abolition Referendum», ob. cit., p. 129.

39 Madison XXXIX, НAmilton/Madison/Jay, El federalista, ob. cit., p. 161.

40 Así, el modelo del Bundesrat alemán, con delegados de los territorios y mandato imperativo, es peculiar y tiene su propia historia. Se creó en 1867, aunque suponía una continuación de la Asamblea Federal establecida en 1815 por la confederación germánica. Vid. LuTHER, J., «The search for a constitutional geography and historiography of second chambers», ob. cit., p. 13. 
infraestatales» ${ }^{41}$. De ahí que, como señalábamos al principio, los Estados federales constituyen una excepción a la tendencia a la baja del bicameralismo durante el siglo $\mathrm{XX}$.

La realidad es que en casi todos los Estados federales existe bicameralismo y actualmente solo hay seis excepciones: los Emiratos Árabes Unidos, Unión de Comoras, Estados Federados de Micronesia, Federación de San Cristóbal y Nieves, Venezuela (se abolió el Senado en 1999) y en Nepal (el National Council se abolió en 2008) ${ }^{42}$.

No obstante, como comprobaremos de los últimos debates sobre el Senado, la más que dudosa función de representación territorial que desempeñan hoy las segundas cámaras en los modelos de descentralización política ha derivado en un intenso debate sobre su posible reforma o supresión. Y es que una cosa es que exista el Senado en los Estados federales y otra es que exista una cámara de representación territorial. Una cosa es que exista Senado y otra es que ese Senado aporte algo al concepto de Estado Federal. O en palabras de Torres del Moral, «una cosa es un hecho constitucional generalizado y otra un principio general del Derecho Constitucional» ${ }^{43}$. En este sentido, autores como Watts excluyen ya el bicameralismo en el concepto de Estado Federal ${ }^{44}$.

La realidad de los cuatro casos que analizamos a continuación no hace sino confirmar lo que tempranamente Carré de Malberg ${ }^{45}$ o Durand ${ }^{46}$ advirtieron: la imposibilidad de una representación territorial y la asimilación del Senado en los Estados Federales a cámaras de representación política mediadas por partidos. Y con ello — veremos — la asimilación de los términos del debate sobre el Senado con los Estados unitarios.

\section{a) El caso canadiense}

Durante las últimas tres décadas se ha discutido en Canadá sobre la reforma o la supresión del Senado. En un sondeo realizado en junio de 2013 se muestra que una mayoría de canadienses —el 90\% — está ya preparada o bien para

41 Les Secondes Chambres en Europe. Complexité parlementaire ou nécessité démocratique?, Strasbourg, Commission européenne de la démocratie par le droit (Commission de Venise), 2006, p. 1. En línea: http://www. venice.coe.int/webforms/documents/default.aspx?pdffile=CDL(2006)059rev-f (consultado el 16.01.2017).

42 COAKLEY, J., «The Strange Revival of Bicameralism», ob. cit. WATTS, R. L., «Federal second chambers compared», Working Paper, 2, 2008, Kingston: Institute of Intergovernmental Relations, Queen's University, p. 1.

43 TORRES DEL MORAL, A., «El Parlamento: monocameralismo y bicameralismo», ob. cit., p. 37.

44 «(T)hat it is inappropriate to regard a bicameral federal legislature as a definitive characteristic of federations». WATTS, R. L. (2008). Federal second chambers compared, ob. cit., p. 1.

45 CARRÉ DE MALBERG, Teoría general del Estado, Fondo de Cultura Económica, México, 1948, reimpresión 2000, p. 120.

46 DURAND, CH., Les États fédéraux. Étude de droit constitutionnel positif, Sirey, Paris, 1930, p. 204. 
reformar el Senado (49\%) o para abolirlo (41\%), siendo solo el $4 \%$ los que apoyan el status quo y el $4 \%$ de indecisos ${ }^{47}$.

La realidad es que la discusión sobre el Senado fue la que más tiempo ocupó en la Conferencia de Quebec de 1864 y se optó deliberadamente por evitar una segunda cámara electa que había dado muchos problemas bajo el Acto de la Unión $(1840)^{48}$. Según la regulación vigente, los senadores canadienses son designados por el gobernador general a propuesta del primer ministro teniendo en cuenta una distribución en cuatro bloques regionales en igualdad de número de senadores. Las autoridades provinciales no intervienen en absoluto en el proceso de nombramiento. $\mathrm{Y}$ el nombramiento discrecional por parte del primer ministro ha tenido como resultado que en el Senado estén representados los dos grandes partidos tradicionales canadienses, el liberal y el conservador, por ser los únicos que han alcanzado el Gobierno fede$\mathrm{ral}^{49}$. Se eligen, por tanto, en clave partidista y su funcionamiento interno también responde a dicha clave, como ha subrayado el primer ministro de la provincia de Saskatchewan, Brad Wall ${ }^{50}$ o así se ha reconocido recientemente por los propios senadores $^{51}$. Sus funciones legislativas son de segunda lectura y están claramente subordinadas a la Cámara de los Comunes, sin ninguna intervención en la investidura del gobierno ni en la exigencia de responsabilidad política del gobierno.

Este Senado ha sido muy criticado doctrinalmente y durante las últimas tres décadas se han sucedido diferentes propuestas de reforma ${ }^{52}$ que pasan desde reformas menores (eliminar los requisitos que son exigidos para la designación ${ }^{53}$ ) hasta democratizarlo y convertirlo en una cámara de «salvaguarda de intereses regionales $»^{54}$, o bien suprimirlo ${ }^{55}$. La falta de acuerdo ha imposibilitado cualquier modificación constitucional, aunque ha propiciado que al menos, de una manera oficiosa, trate de corregirse el déficit democrático en su nombramiento. El caso de

$47 \mathrm{Vid}$. http://www.cbc.ca/news/politics/majority-wants-senate-changed-or-abolished-poll-suggests1.1398046 (consultado el 2.02.2017).

48 WATTS, R. L., «Federal second chambers compared», ob. cit., p. 8.

49 Castellà Andreu, J. Mª, «Canadá: un laboratorio del federalismo», Solozabal, J. J. (ed.), La reforma federal. España y sus siete espejos, Biblioteca Nueva, 2014, pp. 270-277.

50 Wall, B., «Time to consider abolition of the Senate», Canadian Parliamentary Review, 9, 2013, p. 7.

51 Debates of the Senate, vol. 150, núm. 21, Thursday, March 10, 2016, p. 362. En línea: http://publications.gc.ca/collections/collection_2016/sen/Y3-421-21-eng.pdf (consultado el 2.02.2017).

52 En http://reformorabolish.ca/ pueden consultarse desde 2013 hasta seis posiciones respecto al Senado canadiense, con los argumentos a favor y en contra de cada una de las posiciones.

53 Recientemente, Stevenson, R., «Some Suggestions for Incremental Reform of the Senate», Canadian Parliamentary Review, 2016, pp. 19-23. En marzo de 2016 el senador Dennis Glen Patterson introdujo una propuesta de reforma constitucional en este mismo sentido. Vid. Debates of the Senate, vol. 150, $\mathrm{n}^{\circ} 21$, Thursday, March 10, 2016, pp. 364-365.

54 Esta es la postura del Conservative Party. Vid. Programa electoral para las elecciones de 2015: http:// www.conservative.ca/media/documents/Policy-Declaration-Feb-2014.pdf (consultado el 2.02.2017).

55 Esta es la postura del New Democrat Party, que tras las elecciones de octubre de 2015 es la tercera fuerza en la Cámara de los Comunes. Vid. «NDP Leader Tom Mulcair says he'll seek mandate for Senate abolition», CBCNews, 10 de junio de 2015. En línea: http://www.cbc.ca/news/politics/ndp-leader-tom-mulcairsays-he-ll-seek-mandate-for-senate-abolition-1.3107870 (consultado el 2.02.2017). 
Alberta es el más significativo, ya que su Gobierno provincial ha llegado a organizar cuatro elecciones $(1989,1998,2004$ y 2012) y proponer al primer ministro, con éxito, el nombramiento del elegido ${ }^{56}$.

El último proyecto gubernamental de reforma del Senado se introdujo en 2011. Se pretendía la democratización del nombramiento de los senadores. Aunque el primer ministro federal mantenía la potestad de nominación, serían los gobiernos provinciales y los territorios los que presentarían una lista de candidatos elegidos democráticamente por los ciudadanos de cada provincia. No obstante, la Corte Suprema canadiense en la Reference re Senate Reform de 25 de abril de 2014 ha señalado que semejante reforma exigiría una reforma constitucional ordinaria, frente a la agravada que precisaría la supresión de la cámara ${ }^{57}$. En todo caso, la Corte Suprema ha subrayado la exigencia de unas mayorías —o incluso el consenso - para la reforma constitucional del Senado; mayorías que actualmente no está detrás de ninguna de las opciones manejadas por los diferentes partidos políticos. De ahí que el actual primer ministro Justin Trudeau se decante más bien por una vía de hecho, tratando de reducir el carácter partidista de esta cámara y convertir así al Senado en una cámara de carácter técnico y consultivo a través de la designación de independientes ${ }^{58}$.

\section{b) El caso australiano}

Inspirado en el Senado de EEUU, el Senado australiano es una de esas cámaras que el formalismo jurídico presenta con todos los rasgos para incluirla en la nómina de cámaras de representación territorial: igual número de senadores por estado, mandato más duradero que el de los miembros de la Cámara baja y con fuertes competencias legislativas - incluso con la posibilidad de bloquear los presupuestos- No obstante, «el plan concebido por los constituyentes, es decir, crear un Senado que representara a las entidades descentralizadas, se ha desactivado progresivamente» ${ }^{59}$. Casi inmediatamente se impuso a los intereses territoriales la disciplina de partidos. Además, la introducción en 1948 de un sistema proporcional

56 Morton, T., «No statecraft, questionable jurisprudence: How the Supreme Court tried to kill Senate reform», The school of public policy SPP Research Papers, University of Calgary, 8, 21, 2015, p. 1.

$57 \mathrm{Vid}$. https://scc-csc.lexum.com/scc-csc/scc-csc/en/item/13614/index.do (consultado el 2.02.2017). Una crítica a la rigidez de esta interpretación puede verse en MORTON, T., «No statecraft, questionable jurisprudence: How the Supreme Court tried to kill Senate reform», ob. cit., pp. 1-13.

58 Vid. «Meet the 21 new Trudeau-appointed senators», CBCNews, de noviembre de 2016. En línea: http://www.cbc.ca/news/politics/all-new-senators-appointment-1.3837512. Vid. también https://www.liberal.ca/realchange/senate-reform/ (consultado el 2.02.2017). De hecho se ha constituido en marzo de 2016 un grupo de trabajo de senadores independientes que pretende reducir el carácter partidista de la cámara y hacerla más profesional. Vid. Debates of the Senate, vol. 150, núm. 21, Thursday, March 10, 2016, p. 362.

59 Biagi, F., «El federalismo australiano», Tajadura, J./De Miguel Bárcena, J. (eds.), Federalismo del siglo XXI, CEPC, Madrid, 2014, pp. 405. 
para la elección de esta cámara facilitó la entrada de partidos minoritarios sin presencia en la Cámara de representantes. Por ello, independientemente del plan inicial de los constituyentes, el senado australiano, por efecto del sistema electoral y del sistema de partidos, se ha convertido más bien en una cámara de revisión ( «house of review») y control de la mayoría gubernamental, forzada a pactar con los partidos minoritarios para poder sacar adelante la leyes aprobadas en la cámara de representantes ${ }^{60}$.

En esta función de control, sólo en una ocasión en la historia constitucional australiana el Senado llegó a bloquear a la primera cámara forzando la convocatoria de nuevas elecciones. Fue la crisis institucional de 1975. En ese caso, las elecciones de 1974 habían confirmado en el Gobierno al partido laborista dirigido por Gough Whitlam, que no contaba con la mayoría suficiente en el Senado. En 1975 el Senado, dominado por la mayoría liberal-nacional, retrasó la aprobación del presupuesto con el objetivo de forzar la dimisión del primer ministro. Finalmente el gobernador general disolvió el Parlamento y se convocaron nuevas elecciones.

Fue fundamentalmente a raíz de esa crisis institucional cuando se empezaron a plantear posibles propuestas de reforma del Senado para reducir sus poderes. Concretamente ha habido seis propuestas de reforma constitucional del Senado que se han sometido a referéndum. Tres de ellas $(1974,1977,1984)$ intentando hacer coincidir las elecciones de las dos cámaras y tratando, con ello, de evitar mayorías diferentes. Los referéndums de 1977 y 1984 arrojaron una mayoría nacional de votos a favor de la reforma, aunque no se consiguió la mayoría de los Estados $^{61}$. En estos casos parece claro que la mayoría de los electores de los Estados menos poblados (y más beneficiados en la representación senatorial) son los más firmes defensores del status quo. No hay que olvidar que al fin y al cabo la igual representación en el Senado de los Estados con muy diferentes población ha otorgado una posición de poder dentro de los propios partidos a los ciudadanos, grupos de interés y gobiernos de los Estados más pequeños ${ }^{62}$.

Al margen de las propuestas de reforma constitucional sometidas a referéndum, el debate sobre el Senado en Australia se produce básicamente entre los que están a favor de un bicameralismo fuerte frente a los que proponen la reducción de los poderes de esta cámara. Resulta de interés subrayar que la defensa del status quo la realizan fundamentalmente los partidos minoritarios y que no se hace en términos de federalismo y de defensa de una segunda cámara

60 Brenton, S., «State-based Representation and National Policymaking: The Evolution of the Australian Senate and the Federation», The Journal of Legislative Studies, 21, 2, 2015. En línea: http://www.tandfonline.com/doi/abs/10.1080/13572334.2014.979626 (consultado el 7.02.2017).

$61 \mathrm{http}: / /$ www.c2d.ch/votes.php?table=votes (consultada el 6.02.2017).

62 Stone, B., «The australian senate: strong bicameralism resurgent», Luther, J./PAssaglia, P./ TARCHI, R. (eds.), A world of second chambers: Handbook for constitutional studies on bicameralism, Giuffré Editore, 2006, pp. 568, 575. 
de carácter territorial, sino que se hace en términos de control y check and balance para corregir así los impulsos mayoritarios de la primera cámara ${ }^{63}$. Son partidarios, en cambio, de la reducción de sus poderes los dos grandes partidos, abogando el Labor Party incluso por su supresión entre 1919 y $1979^{64}$.

\section{c) El caso italiano}

La fallida reforma constitucional italiana a través del referéndum del 4 de diciembre de 2016 ponía también sobre la mesa el debate sobre el papel de la segunda cámara; un debate que se remonta ya a los mismos debates constituyentes en 1946 y 1947.

En una primera fase del debate constituyente italiano se enfrentaron los defensores de la existencia de una única cámara (comunistas y socialistas) frente a los demócratas cristianos que defendían el bicameralismo. Los argumentos de uno y otro lado son los ya conocidos. Mientras que la izquierda señalaba que la soberanía popular era única y única debía ser su representación, la democracia cristiana advertía de los peligros de una dictadura de asamblea. No obstante, la izquierda pronto terminó por aceptar el bicameralismo y la discrepancia giró en torno a la forma de elección de la segunda cámara y, consiguientemente, sobre su función. Rechazada la propuesta de la democracia cristiana para constituir un órgano de representación de intereses económicos y sociales, y también la idea del Senado como cámara de representación de las regiones con parte de sus miembros nombrados por los consejos regionales, el 7 de octubre de 1947 la Asamblea constituyente aprobó por 190 votos a favor y 181 en contra la elección de los senadores por sufragio universal directo por un sistema uninominal. Finalmente la Constitución italiana entró en vigor en enero de 1948 previendo un sistema bicameral perfecto donde ambas cámaras tenían iguales poderes legislativos y de sanción política al gobierno, siendo además ambas cámaras elegidas por sufragio universal directo. Las únicas diferencias se basaban en el cuerpo electoral (los electores del Senado son los ciudadanos de al menos 25 años, mientas que los del Congreso solo los mayores de 18 años) y de la circunscripción electoral (regional para el Senado, provincial para el Congreso $)^{65}$.

63 En este sentido se defiende el papel del Senado en Gauja, A., «The state of democracy and representation in Australia», Representation, 51, 2015. En línea: http://www.tandfonline.com/doi/ full/10.1080/00344893.2015.1023098? src=recsys (consultado el 7.02.2017); también en BRENTON, S., «State-based Representation and National Policymaking: The Evolution of the Australian Senate and the Federation», ob. cit.

64 Sobre todas estas perspectivas en torno al Senado australiano vid. STONE, B., «The australian senate: strong bicameralism resurgent», ob. cit., pp. 573-582.

65 Lucarelli, A., «Le Sénat italien: suppression ou transformation?», Pouvoirs. Le Sénat pour quoi faire?, 159, 2016, pp. 109-111. 
Este sistema bicameral perfecto fue sometido a críticas desde el comienzo y se han sucedido diversas propuestas de reforma. Las propuestas han girado en torno a tres ideas: la conversión del Senado en una «cámara de las regiones»; la conversión del Senado en cámara de representación de intereses socioprofesionales; la supresión del Senado como cámara de legislación ${ }^{66}$. Pero es en el siglo XXI cuando estas propuestas se han impulsado desde el gobierno y se han considerado más viables.

El primer proyecto de reforma del Senado fue presentado en 2003 por el gobierno de Berlusconi y estaba fuertemente influido por la Liga Norte. En este proyecto se preveía un sistema legislativo con tres entidades: la Cámara de Diputados, dedicada a las leyes de competencia exclusiva del Estado; las asambleas regionales, dedicadas a las leyes de competencia exclusiva de las regiones; y el Senado dedicado a las competencias concurrentes. Este proyecto fue rechazado en referéndum de junio de $2006^{67}$.

El último proyecto ha sido el presentado por el gobierno de Renzi y aprobado en segunda lectura por el Congreso el 11 de enero de 2016 y por el Senado el 20 de enero de 2016. La propuesta de reforma del Senado presentada a referéndum el 4 de diciembre de 2016 suponía una ruptura con el bicameralismo perfecto hasta ahora vigente, dejando al Senado en una posición claramente subordinada a la primera Cámara. La propuesta reducía el número de senadores de 315 a 100, 95 de los cuales serían representantes de las instituciones territoriales (Consejos regionales y ayuntamientos). Excluido de las funciones de confianza del Gobierno, se limitaría a intervenir en plano de igualdad con la Cámara de los Diputados en tasados procedimientos legislativos (art. 70.1 del proyecto), con posibilidad de proponer enmiendas en otros (art. 70.3 y art. 70.4 del proyecto), pero teniendo, en estos casos la última palabra la primera cámara ${ }^{68}$. El bicameralismo hubiera pasado a un cuasi-monocameralismo ${ }^{69}$.

Con una participación del 68,48\%, el 59,95\% de los ciudadanos que fueron a votar rechazaron la reforma. El principal argumento en contra de la reforma era la función de contrapeso del Senado frente a la mayoría de la primera cámara. Los constitucionalistas más reticentes con esta reforma señalaban que la disminución del papel del Senado, unido a que la nueva ley electoral aprobada en mayo de 2015 establece que la lista que supere el 40\% de los votos se quede con el 55\% de los 630 escaños de la Cámara de los Diputados, podría llevar a una «deriva

66 Lucarelli, A., «Le Sénat italien: suppression ou transformation?», ob. cit., pp. 111-113. p. 107.

67 Portelli, H., «Bicamérisme ou pouvoir regional», Pouvoirs. Le Sénat pour quoi faire?, 159, 2016,

68 Página oficial del Ministero dell'Interno. Riforma costituzionale 2016. Il Referendum. En línea: http://elezioni.interno.it/contenuti/report/Il_Dossier_Referendum_costituzionale_4_dicembre_2016.pdf (consultada el 1.02.2017)

69 Portelli, H., «Bicamérisme ou pouvoir regional», ob. cit., p. 107. 
autoritaria» ${ }^{70}$. La reiterada función, por tanto, de check and balance. Lamentablemente dos básicamente fueron los factores que impidieron un debate centrado en el papel del Senado. En primer lugar, la personalización de la campaña del referéndum al ligar el Presidente Renzi su continuidad en el cargo con el resultado del mismo. En segundo lugar, el plantear varias reformas constitucionales conjuntamente y no plantear para cada una de ellas una pregunta por separado. Son, sin duda, dos factores que jugaron en detrimento de la transparencia y de la deliberación que exige todo referéndum y que hubiera exigido la reforma del Senado ${ }^{71}$.

\section{d) El caso belga}

Terminaremos señalando el caso federal en el que sí ha prosperado la reforma del Senado en la línea de disminuir sustancialmente su papel. Es el Senado belga. Hasta la reforma constitucional de 31 de enero de 2014, los senadores belgas eran elegidos directamente por el electorado francófono y neerlandófono e indirectamente por los consejos comunitarios o cooptados por los anteriores. No obstante, el Senado no era una Cámara representativa de las comunidades lingüísticas ni de los entes federados, sino, junto con la Cámara baja, un órgano de representación de la nación, según establece el art. 42 de la Constitución de $1993^{72}$. Tras las elecciones legislativas federales de 2010 se firmó el Acuerdo institucional para la sexta reforma del Estado de 11 de octubre de 2011. El acuerdo contemplaba la reforma del Senado para, según la exposición de motivos, garantizar la participación de los parlamentos de los entes federados en la organización y funcionamiento fundamentales del Estado Federal, así como crear un vínculo real de encuentro para los Parlamentos de Comunidad y Región ${ }^{73}$.

Lo cierto es que en Bélgica el Senado desde hace años es una cámara muy discutida y en la que no han faltado voces que piden su supresión desde las más altas instancias. En esta línea estaba la reforma postulada por el primer ministro Guy Verhofsdat, que contemplaba fusionar la Cámara baja y el Senado en un sola

70 «Las claves de la polémica reforma constitucional en Italia», El Mundo, 4 de diciembre de 2016. En línea: http://www.elmundo.es/internacional/2016/12/04/5840456646163fa9618b45d5.html (consultado el 10.02.2017); «Siete puntos claves para entender el referéndum de Italia», Expansión, 30 de noviembre de 2016. En línea: http://www.expansion.com/economia/politica/2016/11/30/583dda2546163fa6358b45bb. html (consultado el 10.01.2017).

71 SÁenz Royo, E. , «a regulación del referendo en el derecho comparado: aportaciones para el debate en España», REDC, 108, 2016, pp. 137-139.

72 Garrido López, C., «Pero... ¿puede ser el Senado una cámara de representación territorial?», REDC, 107, 2016, p. 98.

73 Ruíz Ruíz, J. J., «Más dependiente y menos competente: el nuevo senado belga de comunidades y regiones tras la reforma constitucional», ob. cit., p. 340. 
Cámara y crear un Consejo federal con funciones limitadas ${ }^{74}$. De hecho, el debate que ha terminado con la modificación constitucional de 2014 era entre «la supresión o la reforma del Senado» ${ }^{75}$.

Tras la reforma aprobada en 2014, el Senado ha ido reduciendo su composición de 184 (hasta 1993) a 60 miembros en la actualidad, 50 de los cuales son delegados de las entidades federadas (procedentes de los parlamentos regionales o comunitarios), pasando a tener un papel esencialmente consultivo en el proceso legislativo, a excepción de la materia constitucional o algunas leyes especiales. Sólo si al menos 31 de los 60 senadores se ponen de acuerdo, podrá el Senado discutir las leyes aprobadas en la primera Cámara. El federalismo y la participación de las partes y de las diferentes comunidades (flamenca, francófona y germanófona) quedan garantizadas a través del incremento de poder de las asambleas regionales y de la puesta en marcha de procedimientos para protección de estas comunidades en la toma de decisiones de la Cámara de representantes ${ }^{76}$.

Con ello el Estado federal belga parece considerar prescindible a la segunda cámara, utilizando otros instrumentos para garantizar la participación de las partes y comunidades y, como señala el constitucionalista Christian Behrendt, «poniendo en el frigo esta institución» que se ha convertido «en la más insignificante del país» ${ }^{77}$. De esta forma, aunque formalmente se mantenga la segunda cámara, el monocameralismo se ha convertido en este Estado federal en la regla general $^{78}$, percibiéndose a la Cámara baja como una mejor instancia para integrar los intereses comunitarios y federalizantes del país ${ }^{79}$

\section{CONSIDERACIONES FINALES}

Incuestionablemente aceptado en EEUU, el Senado ha estado en el siglo xx y también en el siglo XXI permanentemente cuestionado en el resto de Estados democráticos. El repunte del bicameralismo durante el siglo XXI no tiene su correspondencia con la democracia contemporánea, de manera que bicameralismo y democracia siguen siendo dos términos difícilmente encuadrables en la misma ecuación.

74 Ruíz Ruíz, J. J., El veto del Senado, CEPC, 2007, pp. 390-400.

75 «Le Sénat après les élections: un noveau «brol» belge», L'Avenir, 13 de mayo, 2014. En línea: http://www.lavenir.net/cnt/dmf20140513_00475499 (consultado el 1.02.2017).

76 Portelli, H., «Bicamérisme ou pouvoir regional», ob. cit., p. 105.

77 «Le Sénat après les élections: un noveau «brol» belge», L'Avenir, 13 de mayo, 2014. En línea: http://www.lavenir.net/cnt/dmf20140513_00475499 (consultado el 1.02.2017).

78 Ruíz Ruíz, J. J., «Más dependiente y menos competente: el nuevo senado belga de comunidades y regiones tras la reforma constitucional», ob. cit., p. 355.

79 Garrido López, C., «Pero... ¿puede ser el Senado una cámara de representación territorial?», ob. cit., p. 98. 
El debate sobre la supervivencia del Senado ha sido constante desde el siglo $\mathrm{xx}$ en las democracias con estructuras territoriales unitarias. Un debate perpetuo que se prolonga hasta nuestros días y en el que básicamente son dos los argumentos - más bien débiles - que se utilizan para su mantenimiento. Por una parte, su función como cámara de enfriamiento de las pasiones, de reflexión y, en definitiva, de mejora de la técnica legislativa. Por otra parte, su función como posible corrector de los posibles excesos mayoritarios de la primera cámara. Los tres casos más significativos demuestran, no obstante, que más allá de funciones más que discutibles, la garantía de su supervivencia se encuentra en la extrema cautela en la utilización de sus poderes residuales.

Durante las últimas décadas también se observa un intenso debate sobre el futuro del Senado en Estados Federales. La más que dudosa función de «representación territorial», que desde el punto de vista teórico parecía dar suficiente sustento a la segunda cámara en esta forma jurídica de Estados, pero que en la práctica se ha tornado imposible, parece estar en el fondo del cuestionamiento de esta cámara. Y es que la dinámica partidista parece sobreponerse de forma general a cualquier territorialización del voto de los senadores, haciendo de la representación territorial una quimera. Puede que en los Estados Federales las segundas cámaras federales nacieran «en pleno proceso de unificación política como consecuencia de la necesidad lógico-jurídica de expresar unificadamente la diversidad territorial federada, pero dicha lógica ha cedido frente a la dinámica mediatizadora de las mediaciones partidistas y, con ello, el bicameralismo federal ha perdido su objeto» ${ }^{80}$. De ahí que los debates sobre el Senado en los cuatro casos analizados reproduzcan — curiosamente- los mismos argumentos que los utilizados en los Estados unitarios. Frente a la tendencia a una drástica reducción de sus poderes (efectiva ya en Bélgica), la pervivencia de estas segundas cámaras se aferra fundamentalmente a su carácter técnico y más bien consultivo (es la tendencia de facto que pretende Trudeau en Canadá) o a su función como elemento de contrapeso del principio mayoritario (caso de Australia o Italia).

En todo caso, tanto en los Estados unitarios como federales, la supervivencia de la segunda cámara en el siglo XxI exige, como puede deducirse de los últimos debates, o bien un drástico recorte de sus poderes, o bien una utilización cautelosa de los que tiene. No obstante, su existencia formal parece asegurada. Lo cierto es que «todas las segundas cámaras se han establecido, y se han mantenido, no desde un desinteresado amor a la deliberación madura, sino porque hay algo que se pretende defender contra el resto de la comunidad ${ }^{81}$. Y una vez creada resulta muy difícil su supresión, ya que esa cámara dificultará cualquier reforma que no le asegure su propia subsistencia como institución.

80 Garrido López, C., «Pero... ¿puede ser el Senado una cámara de representación territorial?»,ob. cit., p. 99.

81 Finer, H., The theory and practice of modern government, vol. 1, Methuen, London, 1946, p. 677. 
TITLE: Bicameralism in the 21st century. The latest debates on the Senate in comparative law

ABSTRACT: From the moment that liberal revolutions attributed sovereign power to the nation and constituted representative government as the only way to organize the exercise of political power, bicameralism and, in particular, the Senate will become a much discussed institution in comparative law. The extension of suffrage and the assumption of the democratic principle of popular sovereignty will further enhance the contradiction between the single popular will and its double expression. According to this idea, the twentieth century has shown a clear downward trend of bicameralism in the unitary states; not so in federal states. This paper analyzes the trend of bicameralism at the beginning of the 21st century, as well as the most recent debates around the Senate in both unitary and federal states. We will see how, despite the initial impression, the reduction of Senate power is widespread in contemporary democracies.

RESUMEN: Desde que las revoluciones liberales atribuyeran el poder soberano a la nación y se configurara el gobierno representativo como única forma de organizar el ejercicio del poder político, el bicameralismo $y$, en concreto, el Senado se convertirá en una institución muy discutida en el derecho comparado. La extensión del sufragio y la asunción del principio democrático de la soberanía popular todavía harán más palmaría la contradicción entre la voluntad popular única y su expresión doble. De conformidad con esta idea, el siglo XX ha mostrado una clara tendencia a la baja del bicameralismo en los Estados unitarios; no así en los Estados federales. El presente trabajo analiza la tendencia del bicameralismo a comienzos del siglo XXI, así como los más recientes debates en torno al Senado tanto en Estados unitarios como en Estados Federales. Observaremos cómo, pese a la impresión inicial, la reducción del poder del Senado se generaliza en las democracias contemporáneas.

KeY WORDS: Bicameralism. Senate.

Palabras Clave: Bicameralismo. Senado.

FECHA DE RECEPCIÓN: 13.02.2017

FECHA DE ACEPTACIÓN: 26.07.2017 